Optimization and Inverse Problems in Electromagnetism 


\section{Optimization and Inverse Problems in}

\section{Electromagnetism}

Edited by

Marek Rudnicki

Academy of Humanities and Economics,

Lódź, Poland

and

Sławomir Wiak

Academy of Humanities and Economics,

Lódź, Poland

SPRINGER-SCIENCE+BUSINESS MEDIA, B.V. 
A C.I.P. Catalogue record for this book is available from the Library of Congress.

ISBN 978-90-481-6375-5 ISBN 978-94-017-2494-4 (eBook)

DOI 10.1007/978-94-017-2494-4

Printed on acid-free paper

\author{
All Rights Reserved \\ (C) 2003 by Springer Science+Business Media Dordrecht \\ Originally published by Kluwer Academic Publishers in 2003 \\ Softcover reprint of the hardcover 1st edition 2003 \\ No part of this work may be reproduced, stored in a retrieval system, or transmitted \\ in any form or by any means, electronic, mechanical, photocopying, microfilming, recording \\ or otherwise, without written permission from the Publisher, with the exception \\ of any material supplied specifically for the purpose of being entered \\ and executed on a computer system, for exclusive use by the purchaser of the work.
}




\section{Table of Contents}

Editors' Preface

Chapter 1: General Papers - introductory remarks

Chapter 2: Methodologies - introductory remarks

xiii

Chapter 3: Applications - introductory remarks

xvii

Biographies of editors

xxi

\section{General Papers}

How to Find New and Fresh Ideas

T. Jokinen

Mimicking Nature: How Nature Inspires Methodologies in

Computational Electromagnetics

A. Savini

Comparing Stochastic Methods on SMES Optimization

O. Hajji, S. Brisset, P. Brochet

\section{Methodology}

Using Quasi Random Sequences in Genetic Algorithms

H. Maaranen, K. Miettinen, M.M. Mäkelä

Symbolic Treatment on Equation Based Models to Prepare

Code Generation for Optimization Process

L. Allain, L. Gerbaud, C. Van Der Schaeghe

Multiobjective Shape Design in Electromechanics:

A Contribution to Benchmarking

P. Di Barba 
A New Method for Optimizing the Designing of Grounding Grids

G. Delvecchio, M. Castellitti, N. Medoro, M. Sylos Labini and D. Verde

Solving ODE for Optimization: Specific Use of the Matrix Approach

V. Fischer, L. Gerbaud

Use of Discretization and Solution History in Stochastic Optimization

J. Lähteenmäki

A Genetic Algorithm Method for Determining the Maximum

Touch Voltage Generated by a Grounding System

M. Sylos Labini, G. Delvecchio and F. Neri

Optimization Techniques Benefits for Magnetic Component Design

D. Magot, F. Wurtz, B. Cogitore, J.-P. Keradec

Numerical Optimization Methods in Magnet Design

M. Rudnicki, A. Tomczyk, K. Sasiak

The Use of Quasi-red and Quasi-yellow Nonobtuse Refinements in the Solution of 2-D Electromagnetic PDE's

J. Stańdo, S. Korotov, M. Rudnicki and D. Krawczyk-Stańdo

2-D Inverse Problem: Ideal Current Distribution Generating a Magnetic

Force Field with Given Constraints on Homogeneity

L. Quettier, A. Mailfert

S.M.E.S. Optimization Using the Experimental Design Method

S. Vivier, M. Hecquet, F. Gillon, P. Brochet

Optimal Design of Axial Field Silicon Micromotor by Use of

Field/Circuit Models

S. Wiak

Exponential family and inverse problems.

On Metamorphoses of Probability Theory

A. Gottvald

Topological Evolution and Inverse Problems.

On Probability Between Discrete and Continuous

A. Gottvald

Identification of Industrial Electromagnetic Field Sources 


\section{Applications}

Capitalisation and Treatment of Models for the Optimization of Electric Drives L. Allain, L. Gerbaud, C. Van Der Schaeghe

Application of Screening Analysis to the Optimization of an

Electromagnetic Induction Heating Device S. Alfonzetti, E. Dilettoso and N. Salerno

Identification of Conductivity Distribution in A Au-Al Junction

for Application to Integrated Circuits

A. Bramanti, P. Di Barba, A. Savini, U.A. Tamburini and

F. Maglia

Inverse Problem of Voltage Over Electrostatic Shield

Gap in Helical Transformer Windings

P. Bodlovic, K. Hot and T. Kelemen

Mixing of FEM and Analytical Modeling:

Preliminary Design of a Transformer

B. Delinchant, F. Wurtz and J. Fandino

An Evolutionary Algorithm for the Optimal Design of a Rare

Earth Permanent Magnet Machine

Ö. Göl, B. Sobhi-Najafabadi

Optimization of the Force Characteristic of a Solenoid

Electromagnet with Ferromagnetic Disc in the Coil

$V$. Gueorgiev, A. Alexandrov and I. Yatchev

Optimization of Permanent-Magnet DC Motors Using Orthogonal Arrays K. Hot and P. Bodlović

Identification of Field Model's Parameters in the Turbine

Generator End Zone Using Measured Data

I. Iatcheva, R. Stancheva

Permanent Magnet Machine Optimization by using

FEM and Sensitivity Analysis Techniques

G.D. Kalokiris, A.G. Kladas and J.A. Tegopoulos

Optimization Under Constraints of Static Converters.

Application to a Flyback Structure in PFC Mode C. Larouci, J.P.Ferrieux, L.Gerbaud, J.Roudet 
Nondestructive Quality Testing of High Temperature Superconducting

Bulk Material Used in Electrical Machines and Magnetic Bearings R. Palka, H. May, W.-R. Canders

Increasing of Output Power Capability in a Flux-Weakened

Permanent Magnet Synchronous Motor With a Third

Harmonic Current Injection

B. Stumberger, A. Hamler, M. Trlep, M. Jesenik and V. Goričan

A Method of Shortening Breaking Time of the Magnetic

Contactors by the External Magnetic Forces

Hyun-Kil Cho, Eun-Woong Lee 


\section{Editor's Preface}

The Workshop "Optimization and Inverse Problems in Electromagnetism" is a bi-annual event. The history dates back to 1989, Pavia, Italy. The following meetings were held in Warsaw, Poland (1992), Geneva, Switzerland (1994), Brno, Czech republic (1996), Jyväskylä, Finland (1998) and Turin, Italy (2000). The next Workshop will be held in Grenoble, France in 2004. The aim of the Workshop is to discuss recent developments and application in optimization and inverse methodologies for electromagnetic fields. The meeting is intended to be a forum for applied mathematicians, computer scientists and electrical engineers to exchange ideas, experience on the new developments, trends and applications from industrial and academic viewpoints on the topic. Particular attention is paid to optimization of electromagnetic devices and micro-systems along with software development. An important goal of the workshop is also stimulating personal contacts and co-operation, especially between industrial and academic institutions.

In the year 2002, from 12 to 14 September, the Workshop was jointly organised by The Academy of Humanities and Economics (AHE) and the Institute of Mechatronics and Information Systems (previously the Institute of Electrical Machines and Transformers) of the Technical University of Łódź.

The hosting institution, Academy of Humanities and Economics, is the oldest and the largest private university in Łódź, with over 18.000 students. It employs numerous outstanding scientists, academics and expert-practitioners. The Academy of Humanities and Economics participates in the majority of educational programs of the European Union such as Socrates, Leonardo da Vinci and Youth for Europe. AHE is a unique institution offering as well the exceptional course of creativity to all the students, which completes the traditional academic knowledge with additional skills that stimulate individual self-development.

The Institute of Mechatronics and Information Systems conducts teaching on the topic of electrical machines, mechatronics and computer science. It organises numerous scientific conferences, among which the International Symposium on Electromagnetic Fields in Electrical Engineering. The Institute cooperates with Polish Academy of Sciences (PAN), Institute of Electrical and Electronics Engineers (IEEE), International Compumag Society, etc. In the last fifty years the staff of the Institute published approximately 60 monographs and textbooks, about 800 papers, more than 300 scientific-technical expertise.

The contributions selected for the present volume cover a wide spectrum of inverse and optimal electromagnetic methodologies ranging from theoretical to practical applications. This is an outstanding feature of the meeting, which, to our knowledge is the only one focussed on that subject. 
A number of new optimal and inverse methodologies were proposed. There are contributions related to dedicated software. The book consists of three thematic chapters covering:

a General Papers (survey of specific aspects of optimization and inverse problems in electromagnetism),

- Methodologies,

a Industrial Applications.

The book can be useful for students of electrical and electronic engineering, computer science, applied mathematics ( $\mathrm{PhD}$ level) and to researchers interested in this topic.

Prof. Marek Rudnicki

Chairman
Prof. Sławomir Wiak

Scientific Secretary 
PART 1

\section{GENERAL PAPERS}

\section{Introductory remarks}

There are three survey papers in this chapter. The first one entitled :"How to find new and fresh ideas" is written by Tapani Jokinen of Helsinki University of Technology, Finland, The paper deals with how people get ideas and how we can help the team members to cooperate in a group and see the positive sides of other people's ideas. Logical thinking is essentially ineffective in discovering new ideas, and sometimes it even prevents the birth of ideas. When the problem is left out of mind, it is all of a sudden worked out, although we have not given it a thought. Various methods have been developed for idea generation. The problem analysing and solving method, AIR-OPERA, is presented in the paper. Although not related directly to electromagnetic optimization, it provides valuable advice on creative thinking in electrical engineering.

The second paper by Antonio Savini of University of Pavia, Italy, entitled "Mimicking nature. How nature inspires methodologies in computational electromagnetics" may be considered as a survey of methodologies in computational electromagnetics inspired by nature. A brief overview on the attempts to apply mathematical models to investigate nature and, how to deduce mathematical tools from the study of nature is given, Specifically, we observe is a great deal of research work with the ambitious ultimate goal of getting "intelligent" computers, capable of solving problems without explicitly programming them. Moreover, evolutionary computation seems the natural way for programming and implementing bio-computers. From the software viewpoint, new biological processes like that of molecules of the immune systems combating the invading pathogenic micro-organisms are going to stimulate new search methodologies like artificial immune systems. This way, the circle of inspiration from nature is closed. The final author's conclusion is that the book of nature is inherently written in mathematical terms.

The last paper in this chapter entitled "Comparing Stochastic Methods on SMES Optimization" by O. Hajji, S. Brisset and P. Brochet is concerned with the optimization of a superconducting magnetic energy storage device by means of three stochastic methods: genetic algorithms, simulated annealing and tabu search (GA, SA, TS). Three different optimization strategies have been compared on the superconducting magnetic energy storage (SMES) benchmark

On one hand, stochastic methods, explore the search space better than the deterministic ones, but on the other hand, a large number of objective function evaluations is required. To decrease it, a new stop rule for GA, based on the response surface methodology, is proposed. A table of step vectors is applied to accelerate the convergence of SA. For the optimization of the SMES, the improved versions of stochastic algorithm have led to better 
solutions using significantly less time. The parameter sensitivity is studied and improvements are proposed. The paper may be considered as a comparative study of metaheuristic optimization algorithms enlightened on a practical engineering design problem. 
PART 2

\section{METHODOLOGIES}

\section{Introductory remarks}

There is still a widespread and remarkable interest within the electromagnetic community for the refinement of methodologies for field analysis and optimization technique. It is even possible to observe that the implementation of field and optimization theory in the computer codes has contributed to the clarification of the fundamental questions in such discipline like optimization algorithms. The integration of field analysis with optimization techniques has made it possible to move from computer-aided analysis to automated optimal design of the family of electromagnetic devices, electromechanical converters, micromechanical systems (MEMS), etc. Inverse problems actually could be solved, at least in principle, by means of the numerical optimization of an objective function, which depends on many variables and subject to prescribed constraints. Nevertheless, methodologies for optimal design are still complex; moreover the number of fundamental problems are still open. Problems like multiple local minima, large number of unknowns, multiple design criteria, etc. represent the main challenge to the development of computational electromagnetism.

Sixteen papers are included in this chapter; they may be divided into the following subgroups: Genetic Algorithms (1,7), Probability Theory (14,15), Optimization Techniques $(2,3,4,5,6,8,9,10,12,13)$ and Applications $(11,16)$.

The first group of papers is focused on applying genetic algorithms and advanced software to the analysis of:

- Benchmark problems. Studies have been conducted for the use of quasi-random sequences in the initialisation of a genetic algorithm. Sample points in a quasi-random sequence are designed to have very good distribution properties. Solving a large number of continuous benchmark problems from the literature has tested the modified genetic algorithms using quasi-random sequences in the initial population. The numerical results of three genetic algorithm implementations using different quasi-random sequences have been compared to those of a traditional implementation of using pseudo random numbers.

a Grounding system. A new method for quick determining the maximum touch voltage generated by a grounding system leaking a known current is proposed. The touch voltages in the points of the soil surface are calculated by the Maxwell's subarea method and the search for the maximum touch voltage is carried out by an ad hoc genetic program which is based both on the "one-point crossover" technique and on a mutation having a prearranged probability rate.

The second group of papers is devoted to general and specific inverse problems with a structure called Exponential Family:

a Inverse Problems with a structure called Exponential Family. This structure is pivotal in many fundamental theories (Bayesian Probability Theory, Maximum Entropy Principle, Fourier-Laplace Transforms, Generating Functionology, Sufficient Statistics, Lie Groups, ...), and offers extensive applications to Physical Theories and Complex Systems. 
Topology is manifest in Inverse Problems, Electromagnetic Theory and many other fields. Cartan's exterior calculus of differential forms, Lie groups and Exponential Family are central tools for investigating phenomena where some topological changes take place. A fundamental law of topological evolution is equivalent to the First Law of Statistical Thermodynamics, involving a topological interpretation of heat. We link Bayes' Theorem with Triality, D4-group, and Octonions. The proposed inner symmetries of Probability Theory itself correspond to an exceptional A-D-E sequence of Lie groups, with vital implications for fundamental physical laws.

The next group of papers deals with optimization techniques applied to the specific model and technical problems, while the numerical and analytical methods are successfully applied to:

a Symbolic treatment of analytical models. A symbolic treatment of analytical modcls is used to describe systems to be size-using methods with optimization under constraints techniques. Such models are equations based. In many cases, the models have to be arranged by the designer, to get a well organised and oriented description for their valuing in the optimization process. Indeed, a complete physical description for a system is often made of equations, algorithms and functions. To optimise on many criteria, the computer code that values them has to be performed. To provide an automatic help to organise the model elements, and to create a computer science code that carries out the model valuing. The description of these models is stored in a format that will allow easier code generation, for their valuing in different kind of tools, especially in optimization one.

- Pareto criterion for electromechanical converters. The shape synthesis of a linear actuator and a permanent-magnet motor is revisited in terms of multiobjective optimization using Pareto criterion of optimality. Best-compromise solutions are approximated by means of sample-and-rank method. The two case studies are proposed as model problems for inverse electromechanics.

- Choice of automatic grounding grid. A method for choosing, in a quite automatic way, the various grids and so for speeding up the designing process is proposed. The method is based on the determination of the maximum touch voltages, these being calculated by resorting to a genetic algorithm. Moreover, the building cost of the grounding grid is optimized thanks to the "Travelling Salesman" algorithm.

a Gradient optimization algorithms in sizing of electrical devices. The sizing of some electrical devices, mainly electrical circuits, using optimization under constraints, while . more accurately gradient optimization algorithms are proposed.

- Practical improvement of stochastic algorithms with numerical models. Combined use of solution space discretisation and solution history is implemented. discretisation gives the possibility to use a solution history where all the solutions evaluated are stored. Use of the solution history guarantees that a single solution candidate is calculated only once. This approach is useful for the stochastic algorithms that typically evaluate many solution candidates. Then improved method is proposed for optimization of high-speed induction motors modelled with 2D finite element analysis software. 
Finite element method with non-obtuse triangulation. A new refinement techniques called "quasi-red" and "quasi-yellow" are applied for the solution of a non-linear Poisson's equation describing magnetic flux density in 2-D. The equation is solved using the finite element method with non-obtuse triangulation. The main goal is to modify initial mesh in some non-standard way to have solely non-obtuse triangles. Usefulness of the method is demonstrated on the problem of optimal shape design of an electromagnet.

- Experimental Design Method (EDM)/ optimization techniques. Some results on optimization techniques and the Experimental Design Method (EDM) are presented and applied to a superconducting magnetic energy storage (SMES) device. The optimization process is realized through two different approaches. In order to solve easily this problem, an optimization manager based on numerical electromagnetic simulations is employed.

- Silicon disc-type micromotors optimization. The complex field and circuit modeling of silicon disc-type micromotors are proposed. 3-D motor structure is proposed to study. The field analysis and torque calculation results have given the knowledge about the changes of the motor structure in order to increase unit motor torque. Comparative analysis leads to the choice of optimal design of the structure.

- Optimization techniques in magnetic component design. Benefits of optimization techniques applied to magnetic component design are discussed using a comparison between an industrial hand-based design process and a methodology based on constrained optimization techniques. The results show dramatic design time decrease, as well as design process improvements in terms of reliability and formalism, which has to be balanced against encountered difficulties.

a Optimization techniques applied to electromagnet pole-shape design. Two optimization techniques applied to electromagnet pole-shape design are proposed and discussed. The aim was to find a shape, such that a desired magnetic field distribution is achieved in the air-gap. The chosen optimization techniques were simulated annealing and taboo search both applied to the aforementioned problem. For approximate solving of partial differential equations the finite element method was used, while MATLAB with PDE toolbox is exploited.

The last group of papers deals with specific methods and applications. The use of numerical methods are successfully applied to the solution of specific probelms, like:

口 Compensation of the gravity by means of a magnetic force acting on a small diamagnetic volume. Generation of uniform field of magnetic forces in a given domain is a problem with various applications. The main idea is the definition of a way of identifying ideal current distributions able to generate a quasi- uniform force field. Based on one particular expression of the complex magnetic potential, an inverse methodology, which is valid for structures with plane symmetry (2-D field), has been worked out. Currents have been modelled by purely surface (or thin shell) current distributions. 
- Evaluation of the electromagnetic field emitted by an industrial source. The design of a magnetic field shielding apparatus requires simulation both the source and the shield system and evaluation as well the main shield parameters like geometry, materials, position with respect to sources. An identification process of industrial magnetic fields with a negligible harmonic content is performed using a genetic algorithm applied to a simplified source. The proposed source is a suitable combination of three-phase ones and it seems able to create a realistic electromagnetic field distribution both in space and in time. 
PART 3

\section{APPLICATIONS}

\section{Introductory remarks}

A knowledge of electromagnetism is necessary for understanding the operation of electromagnetic devices, electromechanical converters and electronic systems in general, such as sensors, actuators, solid state devices, integrated circuits and micro electro mechanical systems (MEMS); also electromagnetic compatibility represents an increasingly important topic. In general the co-existence of electric, magnetic, thermal and mechanical effects characterizes the global behaviour of any electromagnetic device or system.

There is a quite high degree of attention to applied electromagnetism due to the increasing number of new topics.

We could notice subjects like mechatronics, superconductivity, microelectronics and biotechnologies among emerging fields in which electromagnetism plays an essential role.

The complex geometry of devices and the non-linearity of magnetic circuits of devices represent unfavourable factors for field calculation. A very small air-gap with different number of slots in primary and secondary parts of converter produces high field gradients. Therefore the fine discretisation of the field region and, sometimes, the coupling of different methodologies of analysis are necessary.

Thirteen papers are included in this chapter; they may be divided into the following subgroups: Optimization Techniques of Devices $(2,7,11)$, Drives $(1,6,8,9,10,13)$, Transformers and Special Problems $(3,4,5,12)$.

The first group of papers is focused on applying field theory and advanced software to the analysis of:

口 Induction heating system. The hybrid FEM-DBCI (Finite Element Method - Dirichlet Boundary Condition Iteration) is exploited to evaluate the objective function. In order to reduce the computing time for the whole procedure, Design of Experiments (DOE) techniques are used to screen significant geometric parameters. The optimization procedure is carried out by means of the Hooke-Jeeves algorithm. The results are compared with those obtained by stochastic optimization of the device.

- Solenoid electromagnet force characteristics. The optimization of the solenoid force is carried out by varying the position and the dimensions of the disc and seeking maximal initial force. Finite element method has been used for field analysis and force computation.

- Static converters by means of analytical models and a gradient optimization algorithm. The aim is to carry out a compromise between the computation speed and the result accuracy, which allows treating more criteria in the same optimization process than a numerical or simulation approach. 
This method is applied to optimise the volume of a PFC (Power Factor Correction) flyback converter under EMC (electromagnetic compatibility), loss and thermal constraints. In this way, an analytical model is developed for each optimization aspect. The use of these models in an optimization approach with a gradient algorithm under constraints, specific tools are developed and integrated in an adequate optimization environment. The optimization results are then validated by experiment

The second group of papers is devoted to general and specific problems of drives. For the optimization of electric drives, both simulation and analytical methods have been developed:

- Simulation and optimization tools of electric drive. Simulation and optimization tools are proposed, while a structure description based on XML. Then, it proposes a process and tools to transform them to obtain calculation component. In this way, both dedicated simulation components and dedicated calculation components are obtained. This is applied to the sizing of electric drive using optimization algorithms.

- Rare earth permanent magnet generator optimization. The suitability of evolutionary algorithms for design optimization of electromagnetic devices is discussed. Development and application of such an algorithm for such generator is then proved. Test results for a proof of concept generator are given for verification.

口 Permanent magnet DC motor optimization. The performance of permanent-magnet DC motor can be improved by optimization of its magnetic circuit taking into account important effects of magnetic density distribution in motor air-gap and neutral zone. The optimization can be achieved by means of finite element method in field and torque calculation and statistical analysis using orthogonal arrays. The electromagnetic torque as an objective function was calculated directly from magnetic vector potentials of moving conductors as an average value through a slot pitch shift. A fast improvement in shape of some motor parts was achieved by a very limited number of field and torque calculations. The optimization results verified this approach in modeling of DC motors with permanent magnets.

- Identification of turbine generator parameters. Parameters of the mathematical model of the coupled - electromagnetic and temperature fields have been determined; using measured temperature data on the surface of the flange and fingers in the end zone. Different modes (rated, at the leading power factor and at the lagging power factor) are considered. Coupled field problem - electromagnetic and temperature field distribution in the end zone of a $200 \mathrm{MW}$ turbogenerator is studied using FEM for forward problem. The secondary model of the temperature values at points with measuring control has been obtained using combination of response surface methodology and design of experiments.

- Electrical machine design with new magnetic material characteristics. The materials considered are amorphous alloy ribbons as well as Neodymium alloy permanent magnets involving very low eddy current losses. Such materials enable electric machine operation at higher frequencies compared with the standard iron laminations used in the traditional magnetic circuit construction and provide better efficiency. 
- Improvement in a six-phase flux-weakened permanent magnet synchronous motor with a third harmonic current injection. It is shown that the flux-weakened permanent magnet synchronous motor with the third harmonic current injection is capable of producing more output torque per RMS current flowing through the windings than the same motor supplied with sinusoidal currents. The influence of the iron losses on the OPC and the constantpower speed range (CPSR) is included in the analysis by the posterior iron loss calculation. The improvement of the machine OPC with a third harmonic current injection depends on the level of flux weakening and it is higher at a high level of flux-weakening.

The next group of papers deals with specific methods and applications. Beyond the common use of numerical methods, analytical methods also are successfully applied to the solution of specific probelms, like:

a $A u$ and Al junction in a current carrying contact for application to integrated circuits. The conductivity of the compound is identified by means of a field-inversion technique. The simulation of the growth process of the Au-Al compound relies on the transient field analysis of the current flow through the junction.

- Multi-conductor transmission line model of a helical winding. An analysis of the very fast electromagnetic transients will include the lumped voltage between the edges of the open electrostatic screen, previously found to be responsible for experimentally found anomalous distortion of the travelling waves. Contrary to the former model with distributed voltage and axial symmetry, the new model requires parameters beyond those of classical theories of wave propagation in transformer windings. The additional parameters are found by matching the simulated voltage transients with the experimental results.

- Methodologies of preliminary design of a three-phase transformer. Preliminary design requires low time cost. Although analytical models answer this issue, they brought some loss of quality, as they are subject to many hypotheses. Analytical model can be mixed with numerical ones to improve the modelling quality. The analytical model was used, with an automatic symbolic differentiation. Then FEM evaluation of the leakage reactance is introduced to the analytical model.

- Non-destructive examination method for high temperature superconductors (HTSC's). The scanning of the magnetic field distribution, which is trapped by a superconductor, is done. The identification of the positions, dimensions and orientations of sub - domains within the HTSC has been reduced for the determination of the critical current density $\left(\mathrm{J}_{\mathrm{c}}\right)$. The defined inverse field problem has been solved by use of numerical algorithm based on FEM. 


\section{BIOGRAPHIES OF THE EDITORS}

Prof. Marek Rudnicki was born on July 23, 1951 in Siedlce. In the course of his academic and research career he has been working for numerous institutions, i.e. High School of Agriculture, Polish Academy of Sciences, University of Warsaw, Technical University of Lodz, and lately for the Academy of Humanities and Economics in Lodz, where he cducts lectures on artificial intelligence, optimization, numerical methods, evolutionary computing as well as computer simulation and modeling. His main research interests include evolutionary computing, computational optimization and artificial intelligence. He has been cooperating with numerous research centers in Poland and abroad, i.e. University of Pavia (Italy), National Technical University of Athens (Greece), University of Jyväskylä (Finland), Helsinki University of Technology (Finland), Aalborg University (Denmark) and Technical University of Madrid (Spain). He has taught a number of PhD courses, e.g. International Summer School of Mathematics in Jyväskylä (Finland), International PhD School of Engineering in Aalborg (Denmark) and University of Pavia (Italy). He has a number of affiliations and memberships in scientific bodies, e.g. Polish Society of Applied Electromagnetism, Association of Polish Electrical Engineers, memberships of International Steering Committee of International Workshop on Inverse Problems and Optimal Design in Electrical Engineering, International Editorial Board of International Journal of Electromagnetics and Mechanics and International Organising Committee of Symposium on Micromachines and Servodrives. He is the author and co-author of the following publications: 1 book (Neittaanmaki, P., Rudnicki, M., Savini, A., Inverse Problems and Optimal Design in Electricity and Magnetism, Clarendon Press, Oxford, 1996, 380 pages), over 50 articles in Polish and foreign journals and postconference monographs (COMPEL, International Journal of Applied Electromagnetics in Materials, Nuclear Magnetic Resonance, Wiley \& Sons, Walter de Gruyter and Plenum Press).

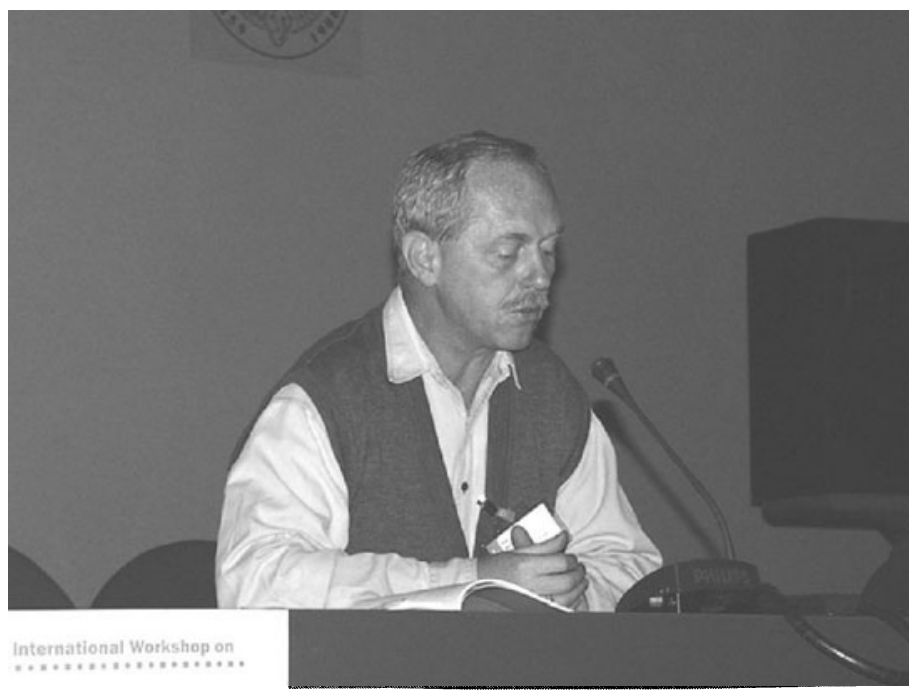


Prof. Slawomir Wiak was born on August 14, 1948. Since the beginning of his academic career he has been working at the Technical University of Lodz. He specializes in technical electrodynamics, computer aided design, computer simulation and modeling, intelligent microsystems modeling, software engineering, databases and expert systems. Not only does he cooperate with numerous research centers all over the world, e.g. University of Southampton (Great Britain), University of Maribor (Slovenia), University of Pavia (Italy), University of West Bohemia (Czech Republic), University of Coimbra (Portugal) and the Czech Academy of Sciences, but also with commercial companies, e.g. Vector Fields Ltd. (Great Britain), where he collaborates with a team of software engineers on computer aided design software. He is an active member of a number of associations and foundations, e.g. IEEE (Institution of Electrical and Electronic Engineers), ICS (International Compumag Society - one of the founders), ICS IEEE (International Computer Society), SEP (Association of Polish Electrical Engineers), Polish Society of Applied Electromagnetism, Polish Society of Theoretical and Applied Electrical Engineering and Śniadeccy Foundation. He is regularly asked to write reviews of articles to be published in the renowned scientific journals, e.g. IEEE Transactions on Magnetism and Elsevier Studies in Applied Electromagnetism in Materials. He is the author and co-author of 3 monographs and scientific dissertations, 9 special issues, 44 articles in foreign journals (e.g. Archiv für Elektrotechnik, IEE, Iternational Journal of Numerical Methods in Engineering, IEEE Transactions on Magnetism and COMPEL), 146 conference papers (presented in COMPUMAG, ISEF, CEFC, IGTE, MIS, ACEMP, ICEM, OIPE). Prof. Wiak is author of over 200 referee reports of papers published in international journals and contributed to International Conferences.

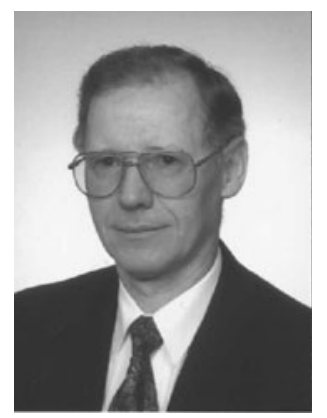

